ADP-96-17/T220

\title{
Self-consistent description of finite nuclei based on a relativistic quark model
}

\author{
Koichi Saito" \\ Physics Division, Tohoku College of Pharmacy \\ Sendai 981, Japan \\ Kazuo Tsushima门 and Anthony W. Thomas \\ Department of Physics and Mathematical Physics, \\ and Institute for Theoretical Physics, \\ University of Adelaide, South Australia 5005, Australia
}

PACS numbers: 12.39.Ba, 21.60.-n, 21.10.-k, 24.85.+p

Keywords: Relativistic mean-field theory, finite nuclei, quark degrees of freedom, MIT bag model, nuclear charge distribution, energy spectra

*ksaito@nucl.phys.tohoku.ac.jp

$\dagger$ †tsushim@physics.adelaide.edu.au

‡athomas@physics.adelaide.edu.au 


\begin{abstract}
Relativistic Hartree equations for spherical nuclei have been derived from a relativistic quark model of the structure of bound nucleons which interact through the (self-consistent) exchange of scalar $(\sigma)$ and vector ( $\omega$ and $\rho$ ) mesons. The coupling constants and the mass of the $\sigma$-meson are determined from the properties of symmetric nuclear matter and the rms charge radius in ${ }^{40} \mathrm{Ca}$. Calculated properties of static, closed-shell nuclei from ${ }^{16} \mathrm{O}$ to ${ }^{208} \mathrm{~Pb}$ are compared with experimental data and with results of Quantum Hadrodynamics (QHD). The dependence of the results on the nucleon size and the quark mass is investigated. Several possible extensions of the model are also discussed.
\end{abstract}




\section{Introduction}

Do quarks play an important role in finite nuclei ? This is one of the central questions in nuclear physics. We now know that explicit quark degrees of freedom are certainly needed to understand deep-inelastic scattering at momentum transfers of several $\mathrm{GeV}$, e.g., the nuclear EMC effect[1]. However, it is perhaps not surprising that such physics seems irrelevant at a scale of a few tens of $\mathrm{MeV}$, and the idea that the quark structure of the nucleon should be of any importance in nuclear physics is often dismissed out of hand.

Within the traditional approach to nuclear physics these tens of $\mathrm{MeV}$ are the result of a fairly fine-tuned cancellation between short-range repulsion, fairly strong intermediate range attraction and large kinetic energy. Nearest neighbour nucleons in nuclear matter tend to be an average $1.8 \mathrm{fm}$ apart, which corresponds to the intermediate range attraction associated with two-pion-exchange. This attraction is mainly generated by the coupling to the $\Delta$ (through the process $\mathrm{NN} \rightarrow \mathrm{N} \Delta \rightarrow \mathrm{NN}$ ), which is just the first excited state of the internal quark structure of the nucleon, namely spin-flip. Of course, one can treat the $\mathrm{NN} \rightarrow \mathrm{N} \Delta$ system in a coupled channels formalism without ever mentioning quark structure. One can even replace the $\mathrm{N} \Delta$ coupling by simple scalar meson $(\sigma)$ exchange without significantly altering the binding energy of nuclear systems. If this were the full story quarks would be irrelevant.

However, it is well known that two-body interactions alone cannot reproduce either the saturation properties of nuclear matter or the binding energies and charge radii of finite nuclei. One needs some form of three-body force, and this leads to a nightmare of badly known form-factors and coupling constants, of inconsistent treatments of the $\pi \mathrm{N}$, NN and NNN systems, and so on 2].

Conventional descriptions of finite nuclei are usually based on the Schrödinger equation which involves nucleons interacting through static potentials. These effective potentials are sometimes determined partly by the Brueckner-Hartree-Fock equations, and often involve some phenomenological dependence on the nuclear density to include the effect of 
higher-order contributions to the N-N force in nuclei[3]. Those density-dependent potentials are however purely phenomenological. Although those conventional approaches give rather successful results for the ground states of nuclei, discrepancies between experimental data and theoretical calculations still remain.

Furthermore, there are several problems at a low energy scale, which might not be explained by the traditional approach. One example is the Okamoto-Nolen-Schiffer (ONS) anomaly [4], which is a well known, long-standing problem in nuclear physics. Conventional nuclear contributions to the anomaly are thought to be at the few percent level and cannot explain the experimental findings[0]. The effects of charge symmetry breaking in the nuclear force, especially $\rho-\omega$ mixing, seem to reduce the discrepancy 团, 囫, 囵. However, recent investigations of the off-shell variation of the $\rho$ - $\omega$ mixing amplitude have put this explanation into question[7].

Another example concerns the unitarity of the Cabibbo-Kobayashi-Maskawa (CKM) matrix elements. There is also a well known discrepancy between the value of the Fermi decay constant extracted from super-allowed Fermi beta-decay of nuclear isotriplets and that required by unitarity of the CKM matrix. This discrepancy remains at the level of a few tenths of a percent (but beyond two standard deviations from the experimental data) after the most rigorous investigation of conventional nuclear and radiative corrections [8].

These facts seem to indicate that the traditional approach may have its limitations and suggest a need for the study of alternate approaches including sub-nucleonic degrees of freedom.

Exciting a single quark in the nucleon costs about $400 \mathrm{MeV}$. This is not significantly different from the energy required to excite a $\Delta$. It is the same order of magnitude as the scalar and vector potentials required in Quantum Hadrodynamics (QHD)[9]. Furthermore, the quarks are very light and should be able to respond rapidly to their environment. We know of no physical argument why this response should be ignored.

About eight years ago Guichon [10 proposed an entirely different model for nuclear matter, based on a mean-field description of nucleons described by the non-overlapping 
MIT bag model, namely the quark-meson coupling (QMC) model. Later this model was refined by Saito and Thomas[11] who clarified its relationship to QHD. Related work has been carried out by a number of groups[12].

The QMC model may be viewed as an extension of QHD in which the nucleons still interact through the exchange of $\sigma$ and $\omega$ mesons. However, the mesons couple not to point-like nucleons but to confined quarks. In studies of infinite nuclear matter [10, 11] it was found that the extra degrees of freedom provided by the internal structure of the nucleon mean that one gets quite an acceptable value for the incompressibility once the coupling constants are chosen to reproduce the correct saturation energy and density for symmetric nuclear matter. This is a significant improvement on QHD at the same level of sophistication. A wonderful feature of this picture is that most specific details of the model are irrelevant. For example, it does not matter that one uses the MIT bag model, all that is needed is that the quarks are relativistic. It does not matter that the $\omega$ generates the repulsion, all that is needed is that it has a vector character.

There have been several interesting applications to the properties of finite nuclei using the local-density approximation. Surprisingly, the model can provide a semi-quantitative explanation of the ONS anomaly when quark mass differences are included[13, [14]. An application of the model, including quark mass differences, has also suggested a previously unknown correction to the extraction of the CKM matrix element, $V_{u d}$, from super-allowed Fermi beta-decay, which would bring the discrepancy in the unitarity problem of the CKM matrix back to only one standard deviation [15, 16]. Furthermore, in the light of current experimental work in relativistic heavy ion collisions [17, 18], which produce nuclear matter at densities several times normal, there has been some initial work on the variation of baryon and meson properties with density[19]. Finally the model has been applied to the case where quark degrees of freedom are undisputedly involved - namely the nuclear EMC effect[20].

However, the inherent problems of the local-density approximation mean that these applications can at best be semi-quantitative and it was clearly very important that the 
extension to finite nuclei be developed. Recently we have succeeded in developing a formulation of the quark-meson coupling model for finite nuclei[21], based on the BornOppenheimer approximation. The effective equation of motion for the nucleon, as well as self-consistent equations for the meson mean fields have been derived. We have also shown some initial results for ${ }^{16} \mathrm{O}$ and ${ }^{40} \mathrm{Ca}$, and discussed, in particular, the spin-orbit force in the model and its relation to the corresponding force in conventional models involving meson exchange between point-like nucleons. Related work has been carried out by several groups[22, 23].

Our aim in this paper is to show details of our calculations for the properties of spherically symmetric, closed-shell nuclei from ${ }^{16} \mathrm{O}$ to ${ }^{208} \mathrm{~Pb}$.

In Sect.2 we briefly summarize the QMC model for both infinite nuclear matter and finite nuclei. Starting from the Lagrangian density proposed in Ref. 21] we derive the Dirac equation for the nucleon and the equations for the meson fields in mean-field approximation and discuss the effective $\sigma$-N coupling constant. The properties of infinite nuclear matter are then reviewed briefly in Sect.2.1. We introduce the couplings of the $\rho$ meson and the photon to the quarks, in Sect.2.2, in order to describe realistic finite nuclei, and again derive explicit forms for the equations of motion of the nucleon and the mesons. In Sect. 3 , the parameters in our calculations are determined so as to reproduce the rms charge radius of ${ }^{40} \mathrm{Ca}$, and then our calculated results are compared with those of QHD and the experimental data. Our results agree with data favorably. We give a summary and discuss some remaining problems and some possible extensions in Sect.⿴囗十

\section{Relativistic formulation of the QMC model}

The solution of the general problem of a composite, quantum particle moving in background scalar and vector fields that vary with position is extremely difficult. One has a chance to solve the particular problem of interest to us, namely light quarks confined in a nucleon which is itself bound in a finite nucleus, only because the nucleon motion is 
relatively slow and the quarks highly relativistic. Thus the Born-Oppenheimer approximation, in which the nucleon internal structure has time to adjust to the local fields, is naturally suited to the problem. It is relatively easy to establish that the method should be reliable at the level of a few percent 21.

Even within the Born-Oppenheimer approximation, the nuclear surface gives rise to external fields that may vary appreciably across the finite size of the nucleon. In Ref. 21], our approach was to start with a classical nucleon and to allow its internal structure (quark wavefunctions and bag radius) to adjust to minimise the energy of three quarks in the ground-state of a system consisting of the bag plus constant scalar and vector fields, with values equal to those at the centre of the nucleon. Of course, the major problem with the MIT bag (as with many other relativistic models of nucleon structure) is that it is difficult to boost. We therefore solve the bag equations in the instantaneous rest frame (IRF) of the nucleon - using a standard Lorentz transformation to find the energy and momentum of the classical nucleon bag in the nuclear rest frame. Having solved the problem using the meson fields at the centre of the "nucleon" (which is a quasi-particle with nucleon quantum numbers), one can use perturbation theory to correct for the variation of the scalar and vector fields across the nucleon bag. In first order perturbation theory only the spatial components of the vector potential give a non-vanishing contribution. (Note that, although in the nuclear rest frame only the time component of the vector field is non-zero, in the IRF of the nucleon there are also non-vanishing spatial components.) This extra term is a correction to the spin-orbit force, which is however very small[21]. Blunden and Miller 23] have also investigated the QMC model for finite nuclei recently. They too reported that the correction due to the variation of meson fields inside the nucleon is not large.

As shown in Ref.[21], the basic result in the QMC model is that, in the scalar $(\sigma)$ and vector $(\omega)$ meson fields, the nucleon behaves essentially as a point-like particle with an effective mass $M_{N}^{\star}$, which depends on the position through only the $\sigma$ field, moving in a vector potential generated by the $\omega$ meson. Because of the vector character, the vector 
interactions have no effect on the nucleon structure except for an overall phase in the wave function, which gives a shift in the nucleon energy.

If we restrict ourselves to consider static, spherically symmetric nuclei, the effective Lagrangian density involving the quark degrees of freedom and the meson fields in meanfield approximation would be given by [21]

$$
\begin{aligned}
\mathcal{L}_{Q M C}^{0} & =\bar{\psi}\left[i \gamma \cdot \partial-M_{N}^{\star}(\sigma(\vec{r}))-g_{\omega} \omega(\vec{r}) \gamma_{0}\right] \psi \\
& -\frac{1}{2}\left[(\nabla \sigma(\vec{r}))^{2}+m_{\sigma}^{2} \sigma(\vec{r})^{2}\right]+\frac{1}{2}\left[(\nabla \omega(\vec{r}))^{2}+m_{\omega}^{2} \omega(\vec{r})^{2}\right]
\end{aligned}
$$

where $\psi(\vec{r}), \sigma(\vec{r})$ and $\omega(\vec{r})$ are respectively the nucleon, $\sigma$ and the time component of $\omega$ fields. (In the mean field approximation, the meson fields are given by their time independent expectation values in the ground state of the nucleus. For a symmetric, parity-eigenstate, the spatial component of the $\omega$ field vanishes.) $m_{\sigma}$ and $m_{\omega}$ are respectively the masses of the $\sigma$ and $\omega$ mesons. $g_{\omega}$ is the $\omega$-N coupling constant (which is related to the corresponding quark- $\omega$ coupling constant, $g_{\omega}^{q}$, as $g_{\omega}=3 g_{\omega}^{q}[10$, 11, 21]).

The effective nucleon mass $M_{N}^{\star}$ is given by a model describing the nucleon structure and it depends on the position through only the $\sigma$ field (as mentioned above). We use the MIT bag model in this paper. (A relativistic oscillator model has also been used in Ref. 23] as an alternative model.) If we define the field-dependent $\sigma$-N coupling constant, $g_{\sigma}(\sigma)$, by

$$
M_{N}^{\star}(\sigma(\vec{r})) \equiv M_{N}-g_{\sigma}(\sigma(\vec{r})) \sigma(\vec{r})
$$

where $M_{N}$ is the free nucleon mass, it is easy to compare with QHD 9]. The Lagrangian density then becomes

$$
\begin{aligned}
\mathcal{L}_{Q M C}^{0} & =\bar{\psi}\left[i \gamma \cdot \partial-M_{N}+g_{\sigma}(\sigma(\vec{r})) \sigma(\vec{r})-g_{\omega} \omega(\vec{r}) \gamma_{0}\right] \psi \\
& -\frac{1}{2}\left[(\nabla \sigma(\vec{r}))^{2}+m_{\sigma}^{2} \sigma(\vec{r})^{2}\right]+\frac{1}{2}\left[(\nabla \omega(\vec{r}))^{2}+m_{\omega}^{2} \omega(\vec{r})^{2}\right]
\end{aligned}
$$

The difference from QHD clearly lies in the fact that the internal structure of the nucleon has forced a known dependence of the $\sigma$-N coupling constant on the scalar field itself. (Note that this dependence is not the same as that adopted in the density-dependent 
hadron field theory 24] where the meson-nucleon vertices are assumed to depend directly on the baryonic densities.)

Equation (11) yields the Dirac equation for the nucleon

$$
\left[i \gamma \cdot \partial-M_{N}^{\star}(\sigma)-g_{\omega} \gamma_{0} \omega\right] \psi=0
$$

as well as the equations for the meson mean-fields

$$
\begin{aligned}
\left(-\nabla_{r}^{2}+m_{\sigma}^{2}\right) \sigma(\vec{r}) & =-\left(\frac{\partial}{\partial \sigma} M_{N}^{\star}(\sigma)\right) \rho_{s}(\vec{r}), \\
\left(-\nabla_{r}^{2}+m_{\omega}^{2}\right) \omega(\vec{r}) & =g_{\omega} \rho_{B}(\vec{r}),
\end{aligned}
$$

where $\rho_{s}$ and $\rho_{B}$ are respectively the scalar and baryon densities of the nucleon in the nucleus, $A$, which are defined by the expectation values in the ground state of $A$ :

$$
\rho_{s}(\vec{r})=\langle A|\bar{\psi} \psi(\vec{r})| A\rangle \text { and } \rho_{B}(\vec{r})=\left\langle A\left|\psi^{\dagger} \psi(\vec{r})\right| A\right\rangle .
$$

One can easily see that the derivative of $M_{N}^{\star}$ with respect to $\sigma$ in Eq.(5) is the response of the nucleon to the external scalar field, and that it is given by the scalar density of a quark in the nucleon[11]:

$$
\left(\frac{\partial M_{N}^{\star}}{\partial \sigma}\right)=-3 g_{\sigma}^{q} \int_{b a g} d \vec{r} \bar{\psi}_{q} \psi_{q} \equiv-3 g_{\sigma}^{q} S(\sigma)
$$

where $g_{\sigma}^{q}$ is the quark- $\sigma$ coupling constant and $\psi_{q}$ is the quark wave function in the nucleon bag with radius $R_{B}^{\star}$ in matter (we denote the bag radius of the free nucleon by $R_{B}$ ). We define $S(\sigma)$ by Eq.(8), which is a function of scalar field itself. Using the MIT bag model[21], $S$ is explicitly given by

$$
S(\sigma(\vec{r}))=\frac{\Omega / 2+m_{q}^{\star} R_{B}^{\star}(\Omega-1)}{\Omega(\Omega-1)+m_{q}^{\star} R_{B}^{\star} / 2},
$$

where $\Omega=\sqrt{x^{2}+\left(R_{B}^{\star} m_{q}^{\star}\right)^{2}}$ is the kinetic energy of the quark and $m_{q}^{\star}(\vec{r})$ is the effective quark mass defined by $m_{q}-g_{\sigma}^{q} \sigma(\vec{r})$, with the quark mass $m_{q}$ inside the free nucleon. Furthermore, we define the scalar density ratio, $S(\sigma) / S(0)$, by $C(\sigma)$ and the $\sigma$-N coupling constant at $\sigma=0$ by $g_{\sigma}$ (i.e., $g_{\sigma} \equiv g_{\sigma}(\sigma=0)$ )

$$
C(\sigma)=S(\sigma) / S(0) \text { and } g_{\sigma}=3 g_{\sigma}^{q} S(0) .
$$

$\S$ Note the change in notation from the earlier works of Saito and Thomas where $C$ was used for what we now call $S$. 
Comparing with Eq.(2), we find

$$
\left(\frac{\partial M_{N}^{\star}}{\partial \sigma}\right)=-g_{\sigma} C(\sigma)=-\frac{\partial}{\partial \sigma}\left[g_{\sigma}(\sigma) \sigma\right],
$$

and the equation for the $\sigma$ field becomes

$$
\left(-\nabla_{r}^{2}+m_{\sigma}^{2}\right) \sigma(\vec{r})=g_{\sigma} C(\sigma) \rho_{s}(\vec{r})
$$

We will discuss the $\sigma-\mathrm{N}$ coupling constant further in the next subsection.

\subsection{Nuclear matter limit}

In isospin-symmetric, infinite nuclear matter the sources of the fields are constant and can be related to the nucleon Fermi momentum $k_{F}[9]$ :

$$
\begin{aligned}
\rho_{B} & =\frac{4}{(2 \pi)^{3}} \int d \vec{k} \theta\left(k_{F}-k\right)=\frac{2 k_{F}^{3}}{3 \pi^{2}} \\
\rho_{s} & =\frac{4}{(2 \pi)^{3}} \int d \vec{k} \theta\left(k_{F}-k\right) \frac{M_{N}^{\star}}{\sqrt{M_{N}^{\star 2}+\vec{k}^{2}}}
\end{aligned}
$$

where $M_{N}^{\star}$ means the constant value of the effective nucleon mass given by the MIT bag model (detailed derivations are given in Ref. 21]).

As in Ref. 21], the bag constant $B$ and the parameter $z_{0}$ (which accounts for the sum of the c.m. and gluon fluctuation corrections) in the familiar form of the MIT bag model Lagrangian are fixed to reproduce the free nucleon mass $\left(M_{N}=939 \mathrm{MeV}\right)$ under the stationary condition in terms of the free bag radius, $R_{B}$. In the following we treat the

free bag radius as a variable parameter to test the sensitivity of our results to the free nucleon size. The results for $B$ and $z_{0}$ are shown in Table 1. We choose the free quark $\operatorname{mass} m_{q}=0,5,10 \mathrm{MeV}$.

Let $(\bar{\sigma}, \bar{\omega})$ be the constant mean-values of the meson fields. From Eqs.([) and (12) we find

$$
\begin{aligned}
\bar{\omega} & =\frac{g_{\omega} \rho_{B}}{m_{\omega}^{2}} \\
\bar{\sigma} & =\frac{g_{\sigma}}{m_{\sigma}^{2}} C(\bar{\sigma}) \frac{4}{(2 \pi)^{3}} \int d \vec{k} \theta\left(k_{F}-k\right) \frac{M_{N}^{\star}}{\sqrt{M_{N}^{\star 2}+\vec{k}^{2}}}
\end{aligned}
$$


Table 1: Bag constant and parameter $z_{0}$ for several bag radii and quark masses.

\begin{tabular}{c|ccc|ccc|ccc}
\hline$m_{q}(\mathrm{MeV})$ & \multicolumn{3}{|c|}{0} & \multicolumn{3}{|c|}{5} & \multicolumn{3}{|c}{10} \\
\hline$R_{B}(\mathrm{fm})$ & 0.6 & 0.8 & 1.0 & 0.6 & 0.8 & 1.0 & 0.6 & 0.8 & 1.0 \\
\hline$B^{1 / 4}(\mathrm{MeV})$ & 211.3 & 170.3 & 144.1 & 210.9 & 170.0 & 143.8 & 210.5 & 169.6 & 143.5 \\
$z_{0}$ & 3.987 & 3.273 & 2.559 & 4.003 & 3.295 & 2.587 & 4.020 & 3.317 & 2.614 \\
\hline
\end{tabular}

where $C(\bar{\sigma})$ is now the constant value of $C$ in the scalar field. This self-consistency equation for $\bar{\sigma}$ is the same as that in QHD, except that in the latter model one has $C(\bar{\sigma})=1[11$, 23]. (This corresponds to the heavy-quark mass limit of the QMC model.)

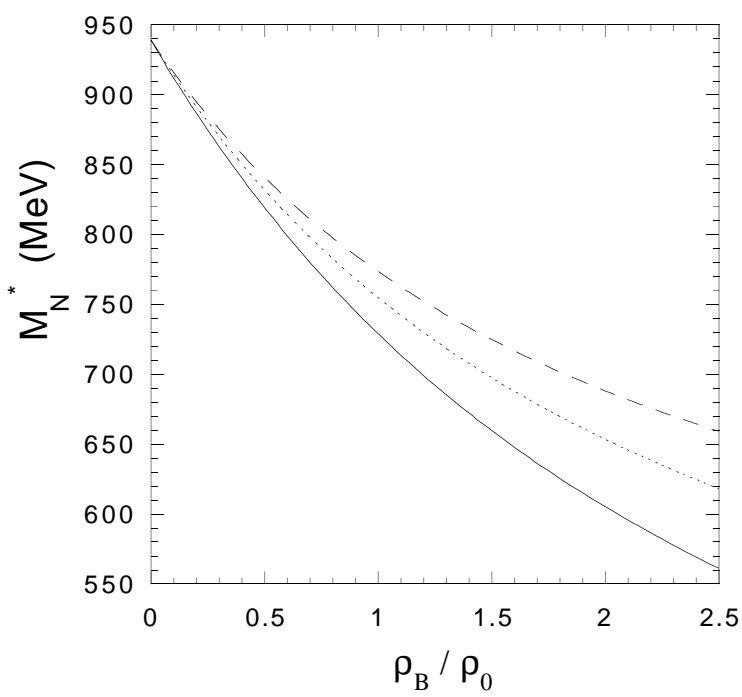

Figure 1: Effective nucleon mass as a function of density (for $m_{q}=5 \mathrm{MeV}$ ). The solid, dotted and dashed curves correspond to $R_{B}=0.6,0.8$ and $1.0 \mathrm{fm}$, respectively.

Once the self-consistency equation for $\bar{\sigma}$ has been solved, one can evaluate the total energy per nucleon 110, 11, 21]:

$$
E^{\text {total }} / A=\frac{4}{(2 \pi)^{3} \rho_{B}} \int d \vec{k} \theta\left(k_{F}-k\right) \sqrt{M_{N}^{\star 2}+\vec{k}^{2}}+\frac{m_{\sigma}^{2} \bar{\sigma}^{2}}{2 \rho_{B}}+\frac{g_{\omega}^{2} \rho_{B}}{2 m_{\omega}^{2}} .
$$

We determine the coupling constants, $g_{\sigma}$ and $g_{\omega}$, so as to fit the binding energy $(-15.7$ $\mathrm{MeV})$ per nucleon and the saturation density, $\rho_{0}=0.15 \mathrm{fm}^{-3}\left(k_{F}^{0}=1.305 \mathrm{fm}^{-1}\right)$, for 
symmetric nuclear matter. The coupling constants and some calculated properties of nuclear matter (for $m_{\sigma}=550 \mathrm{MeV}$ and $m_{\omega}=783 \mathrm{MeV}$ ) at the saturation density are listed in Table 2. The last three columns show the relative changes (from their values at zero density) of the bag radius $\left(\delta R_{B}^{\star} / R_{B}\right)$, the lowest eigenvalue $\left(\delta x / x_{0}\right)$ and the rms radius of the nucleon calculated by the quark wave function $\left(\delta r_{q}^{\star} / r_{q}\right)$ at saturation density.

Table 2: Coupling constants and calculated properties for symmetric nuclear matter at normal nuclear density. The effective nucleon mass, $M_{N}^{\star}$, and the nuclear incompressibility, $K$, are quoted in $\mathrm{MeV}$. The bottom row is for QHD.

\begin{tabular}{c|cccccccc}
\hline$m_{q}(\mathrm{MeV})$ & $R_{B}(\mathrm{fm})$ & $g_{\sigma}^{2} / 4 \pi$ & $g_{\omega}^{2} / 4 \pi$ & $M_{N}^{\star}$ & $K$ & $\delta R_{B}^{\star} / R_{B}$ & $\delta x / x_{0}$ & $\delta r_{q}^{\star} / r_{q}$ \\
\hline \multirow{3}{*}{0} & 0.6 & 5.84 & 6.29 & 730 & 293 & -0.02 & -0.13 & 0.01 \\
& 0.8 & 5.38 & 5.26 & 756 & 278 & -0.02 & -0.16 & 0.02 \\
& 1.0 & 5.04 & 4.50 & 774 & 266 & -0.02 & -0.19 & 0.02 \\
\hline \multirow{5}{*}{5} & 0.6 & 5.86 & 6.34 & 729 & 295 & -0.02 & -0.13 & 0.01 \\
& 0.8 & 5.40 & 5.31 & 754 & 280 & -0.02 & -0.16 & 0.02 \\
& 1.0 & 5.07 & 4.56 & 773 & 267 & -0.02 & -0.19 & 0.02 \\
\hline \multirow{3}{*}{10} & 0.6 & 5.87 & 6.37 & 728 & 295 & -0.02 & -0.13 & 0.02 \\
& 0.8 & 5.42 & 5.36 & 753 & 281 & -0.02 & -0.16 & 0.02 \\
& 1.0 & 5.09 & 4.62 & 772 & 269 & -0.02 & -0.18 & 0.03 \\
\hline \multirow{2}{*}{ QHD } & & 7.29 & 10.8 & 522 & 540 & & & \\
\hline
\end{tabular}

The most notable fact is that the calculated incompressibility, $K$, is well within the experimental range: $K=200 \sim 300 \mathrm{MeV}$. Also our effective nucleon mass is much larger than in the case of QHD. Although the bag radius shrinks a little at finite density, the rms radius of the quark wavefunction actually increases by a few percent at saturation density. 
In Fig.11, we show the effective nucleon mass in medium, as a function of density, for $m_{q}=5 \mathrm{MeV}$. Its dependence on the quark mass is weak. The scalar density ratio $C(\bar{\sigma})$ decreases linearly (to a very good approximation) with $g_{\sigma} \bar{\sigma}[21$. It is very useful to have a simple parametrization for $C$ and the form:

$$
C(\bar{\sigma})=1-a \times\left(g_{\sigma} \bar{\sigma}\right),
$$

with $g_{\sigma} \bar{\sigma}$ in $\mathrm{MeV}\left(\right.$ recall $\left.g_{\sigma}=g_{\sigma}(\sigma=0)\right)$ and $a=(6.6,8.8,11) \times 10^{-4}$ for $R_{B}=(0.6$, $0.8,1.0) \mathrm{fm}$, respectively, is quite accurate up to $2 \sim 3 \rho_{0}$. (These values for the slope parameter, $a$, are valid for all quark masses listed.)

As a practical matter, it is easy to solve Eq.(11) for $g_{\sigma}(\sigma)$ in the case where $C(\sigma)$ is linear in $g_{\sigma} \bar{\sigma}$ as Eq.(18). In fact, we find

$$
M_{N}^{\star}=M_{N}-g_{\sigma}\left[1-\frac{a}{2}\left(g_{\sigma} \bar{\sigma}\right)\right] \bar{\sigma} .
$$

The effective $\sigma$-N coupling constant, $g_{\sigma}(\sigma)$, decreases at half the rate of $C(\sigma)$. Eq.(19) is quite accurate up to twice nuclear matter density.

Having explicitly solved the nuclear matter problem by self-consistently solving for the quark wave functions in the bag in the mean scalar field, one can solve for the properties of finite nuclei without explicit reference to the internal structure of the nucleon. All one needs is Eqs.(18) and (19) for $C(\sigma)$ and $M_{N}^{\star}$ as a function of $g_{\sigma} \bar{\sigma}$. 


\section{$2.2 \quad$ Finite nuclei}

To describe a nucleus with different numbers of protons and neutrons $(Z \neq N)$, it is necessary to consider the contributions of the $\rho$ meson. Any realistic treatment of nuclear structure also requires that one introduces the Coulomb force. The interaction Lagrangian density for the $\rho$ - and $\gamma$-quark couplings in the nucleon IRF is given by

$$
\mathcal{L}_{I}^{\rho+\gamma}=-g_{\rho}^{q} \bar{q}^{\prime} \frac{\tau_{\alpha}^{q}}{2} \gamma_{\mu} q^{\prime} \rho_{\alpha, I R F}^{\mu}-e \bar{q}^{\prime}\left(\frac{1}{6}+\frac{\tau_{3}^{q}}{2}\right) \gamma_{\mu} q^{\prime} A_{I R F}^{\mu}
$$

where $q$ is the quark field (the prime means the IRF) and $\rho_{\alpha, I R F}^{\mu}$ and $A_{I R F}^{\mu}$ are respectively the $\rho$ meson field with isospin component $\alpha$ and the photon field. $\tau_{\alpha}^{q} / 2$ is the isospin operator for the quarks. $g_{\rho}^{q}$ and $e$ are the quark- $\rho$ coupling constant and the electric charge.

We saw in Ref.[21] that this leads to both a central and a spin-orbit potential for the nucleon in the nuclear rest frame. For the isoscalar, $\omega$ meson these potentials are well represented by a vector $\omega$-nucleon coupling because the isoscalar magnetic moment of the nucleon is near unity. However, for the $\rho$, the relativistic formulation at the nucleon level requires a strong tensor coupling $\left(\sim \sigma^{\mu \nu} q_{\nu}\right)$ if it is to reproduce the interaction given by the quark model 21].

In Hartree approximation a tensor coupling gives only a spin-orbit force for a nucleon bound in a static, spherical nucleus. However, in Hartree-Fock it can also give rise to a central force which would be necessary to reproduce the bulk symmetry energy. For simplicity, in the present work we follow the usual procedure adopted in the Hartree treatment of QHD of using only a vector coupling for the $\rho$, with $g_{\rho}$ adjusted to give the bulk symmetry energy in MFA - where $g_{\rho}=g_{\rho}^{q}$. (The accuracy of this approximation will be examined in detail in future work.)

In this case, in mean field approximation, only $\alpha=3$ contributes in Eq.(20). If we denote by $b(\vec{r})$ and $A(\vec{r})$ the mean values of the time component of $\rho$ field and the Coulomb field in the nuclear rest frame, we can transpose our results for the $\omega$ field by trivial isospin 
factors:

$$
3 g_{\omega}^{q} \omega(\vec{r}) \rightarrow g_{\rho} \frac{\tau_{3}^{N}}{2} b(\vec{r}) \quad \text { or } \frac{e}{2}\left(1+\tau_{3}^{N}\right) A(\vec{r})
$$

where $\tau_{3}^{N} / 2$ is the third component of the nucleon isospin operator.

In summary, our effective Lagrangian density in mean field approximation takes the form:

$$
\begin{aligned}
\mathcal{L}_{Q M C} & =\bar{\psi}\left[i \gamma \cdot \partial-M_{N}+g_{\sigma}(\sigma(\vec{r})) \sigma(\vec{r})-g_{\omega} \omega(\vec{r}) \gamma_{0}\right. \\
& \left.-g_{\rho} \frac{\tau_{3}^{N}}{2} b(\vec{r}) \gamma_{0}-\frac{e}{2}\left(1+\tau_{3}^{N}\right) A(\vec{r}) \gamma_{0}\right] \psi \\
& -\frac{1}{2}\left[(\nabla \sigma(\vec{r}))^{2}+m_{\sigma}^{2} \sigma(\vec{r})^{2}\right]+\frac{1}{2}\left[(\nabla \omega(\vec{r}))^{2}+m_{\omega}^{2} \omega(\vec{r})^{2}\right] \\
& +\frac{1}{2}\left[(\nabla b(\vec{r}))^{2}+m_{\rho}^{2} b(\vec{r})^{2}\right]+\frac{1}{2}(\nabla A(\vec{r}))^{2} .
\end{aligned}
$$

The variation of the Lagrangian results in the following equations for static, spherically symmetric nuclei (see also Eqs.(6) and (12)):

$$
\begin{aligned}
\frac{d^{2}}{d r^{2}} \sigma(r)+\frac{2}{r} \frac{d}{d r} \sigma(r)-m_{\sigma}^{2} \sigma(r) & =-g_{\sigma} C(\sigma(r)) \rho_{s}(r) \\
& \equiv-g_{\sigma} C(\sigma(r)) \sum_{\alpha}^{o c c} d_{\alpha}(r)\left(\left|G_{\alpha}(r)\right|^{2}-\left|F_{\alpha}(r)\right|^{2}\right), \\
\frac{d^{2}}{d r^{2}} \omega(r)+\frac{2}{r} \frac{d}{d r} \omega(r)-m_{\omega}^{2} \omega(r) & =-g_{\omega} \rho_{B}(r) \\
& \equiv-g_{\omega} \sum_{\alpha}^{o c c} d_{\alpha}(r)\left(\left|G_{\alpha}(r)\right|^{2}+\left|F_{\alpha}(r)\right|^{2}\right), \\
\frac{d^{2}}{d r^{2}} b(r)+\frac{2}{r} \frac{d}{d r} b(r)-m_{\rho}^{2} b(r) & =-\frac{g_{\rho}}{2} \rho_{3}(r) \\
& \equiv-\frac{g_{\rho}}{2} \sum_{\alpha}^{o c c} d_{\alpha}(r)(-)^{t_{\alpha}-1 / 2}\left(\left|G_{\alpha}(r)\right|^{2}+\left|F_{\alpha}(r)\right|^{2}\right), \\
\frac{d^{2}}{d r^{2}} A(r)+\frac{2}{r} \frac{d}{d r} A(r) & =-e \rho_{p}(r) \\
& \equiv-e \sum_{\alpha}^{o c c} d_{\alpha}(r)\left(t_{\alpha}+\frac{1}{2}\right)\left(\left|G_{\alpha}(r)\right|^{2}+\left|F_{\alpha}(r)\right|^{2}\right),
\end{aligned}
$$

where $d_{\alpha}(r)=\left(2 j_{\alpha}+1\right) / 4 \pi r^{2}$ and

$$
\begin{aligned}
\frac{d}{d r} G_{\alpha}(r)+\frac{\kappa}{r} G_{\alpha}(r)-\left[\epsilon_{\alpha}-g_{\omega} \omega(r)-t_{\alpha} g_{\rho} b(r)\right. & -\left(t_{\alpha}+\frac{1}{2}\right) e A(r)+M_{N} \\
& \left.-g_{\sigma}(\sigma(r)) \sigma(r)\right] F_{\alpha}(r)=0, \\
\frac{d}{d r} F_{\alpha}(r)-\frac{\kappa}{r} F_{\alpha}(r)+\left[\epsilon_{\alpha}-g_{\omega} \omega(r)-t_{\alpha} g_{\rho} b(r)\right. & -\left(t_{\alpha}+\frac{1}{2}\right) e A(r)-M_{N} \\
& \left.+g_{\sigma}(\sigma(r)) \sigma(r)\right] G_{\alpha}(r)=0 .
\end{aligned}
$$


Here $i G_{\alpha}(r) / r$ and $-F_{\alpha}(r) / r$ are respectively the radial part of the upper and lower components of the solution to the Dirac equation for the nucleon ( $\alpha$ labelling the quantum numbers and $\epsilon_{\alpha}$ being the energy) under the normalization condition:

$$
\int d r\left(\left|G_{\alpha}(r)\right|^{2}+\left|F_{\alpha}(r)\right|^{2}\right)=1
$$

As usual, $\kappa$ specifies the angular quantum numbers and $t_{\alpha}$ the eigenvalue of the isospin operator $\tau_{3}^{N} / 2$. $C(\sigma)$ and $g_{\sigma}(\sigma)$ are given by Eqs.(9) and (2), respectively - or practically, by Eqs.(18) and (19), i.e.,

$$
g_{\sigma}(\sigma(\vec{r}))=g_{\sigma}\left[1-\frac{a}{2} g_{\sigma} \sigma(\vec{r})\right]
$$

The total energy of the system is then given by

$$
\begin{aligned}
E_{t o t} & =\sum_{\alpha}^{o c c}\left(2 j_{\alpha}+1\right) \epsilon_{\alpha}-\frac{1}{2} \int d \vec{r}\left[-g_{\sigma} C(\sigma(r)) \sigma(r) \rho_{s}(r)\right. \\
& \left.+g_{\omega} \omega(r) \rho_{B}(r)+\frac{1}{2} g_{\rho} b(r) \rho_{3}(r)+e A(r) \rho_{p}(r)\right] .
\end{aligned}
$$

\section{$3 \quad$ Numerical results for finite nuclei}

Equations (23) to (29) give a set of coupled non-linear differential equations, which may be solved by a standard iteration procedure[25]. In this paper the numerical calculation was carried out by modifying the technique described by Horowitz et al. [25, 26]. Changing the initial condition for the meson fields, the $\sigma-\mathrm{N}$ coupling constant and the scalar density in their fortran program [26], the calculation can be performed very easily" The calculation is achieved in at most 20 iterations when it is performed with a maximum radius of 12 (15) fm on a mesh of $0.04 \mathrm{fm}$ for medium mass $(\mathrm{Pb})$ nuclei.

\footnotetext{
IIf the numerical convergence is slow it may be improved by mixing appropriately the meson potentials given by the $i$-th iteration and those by the $(i-1)$-th iteration - as is usually done in non-relativistic calculations.
} 


\subsection{Determination of parameters}

There are seven parameters to be determined: $g_{\sigma}, g_{\omega}, g_{\rho}, e, m_{\sigma}, m_{\omega}$ and $m_{\rho}$. We take the experimental values: $m_{\omega}=783 \mathrm{MeV}, m_{\rho}=770 \mathrm{MeV}$ and $e^{2} / 4 \pi=1 / 137.036$. The coupling constants $g_{\sigma}$ and $g_{\omega}$ are fixed to describe the nuclear matter properties with $m_{\sigma}$ $=550 \mathrm{MeV}$ in Sec.2.1 (see Table 2).

The $\sigma$-meson mass however determines the range of the attractive interaction and changes in $m_{\sigma}$ affect the nuclear-surface slope and its thickness. Therefore, as in the paper of Horowitz and Serot[25], we adjust $m_{\sigma}$ to fit the rms charge radius of ${ }^{40} \mathrm{Ca}$, $r_{c h}\left({ }^{40} \mathrm{Ca}\right)=3.48 \mathrm{fm}$, the experimental value 27. We should notice here that variations of $m_{\sigma}$ at fixed $\left(g_{\sigma} / m_{\sigma}\right)$ have no effect on the infinite nuclear matter properties. Therefore, keeping the ratio $\left(g_{\sigma} / m_{\sigma}\right)$ constant we vary $m_{\sigma}$ to fit the rms charge radius of ${ }^{40} \mathrm{Ca}$. We expect that $m_{\sigma}$ ranges around $400 \sim 550 \mathrm{MeV}\left[23\right.$, 28]. The last parameter, $g_{\rho}$, is adjusted to yield the bulk symmetry energy per baryon of $35 \mathrm{MeV} 25$. We summarize the parameters in Table 3 .

Table 3: Model parameters for finite nuclei.

\begin{tabular}{cccccc}
\hline$m_{q}(\mathrm{MeV})$ & $R_{B}(\mathrm{fm})$ & $g_{\sigma}^{2} / 4 \pi$ & $g_{\omega}^{2} / 4 \pi$ & $g_{\rho}^{2} / 4 \pi$ & $m_{\sigma}(\mathrm{MeV})$ \\
\hline \multirow{2}{*}{0} & 0.6 & 3.55 & 6.29 & 6.79 & 429 \\
& 0.8 & 2.94 & 5.26 & 6.93 & 407 \\
& 1.0 & 2.51 & 4.50 & 7.03 & 388 \\
\hline \multirow{2}{*}{5} & 0.6 & 3.68 & 6.34 & 6.78 & 436 \\
& 0.8 & 3.12 & 5.31 & 6.93 & 418 \\
& 1.0 & 2.69 & 4.56 & 7.02 & 401 \\
\hline \multirow{2}{*}{10} & 0.6 & 3.81 & 6.37 & 6.78 & 443 \\
& 0.8 & 3.28 & 5.36 & 6.92 & 428 \\
& 1.0 & 2.91 & 4.62 & 7.02 & 416 \\
\hline
\end{tabular}




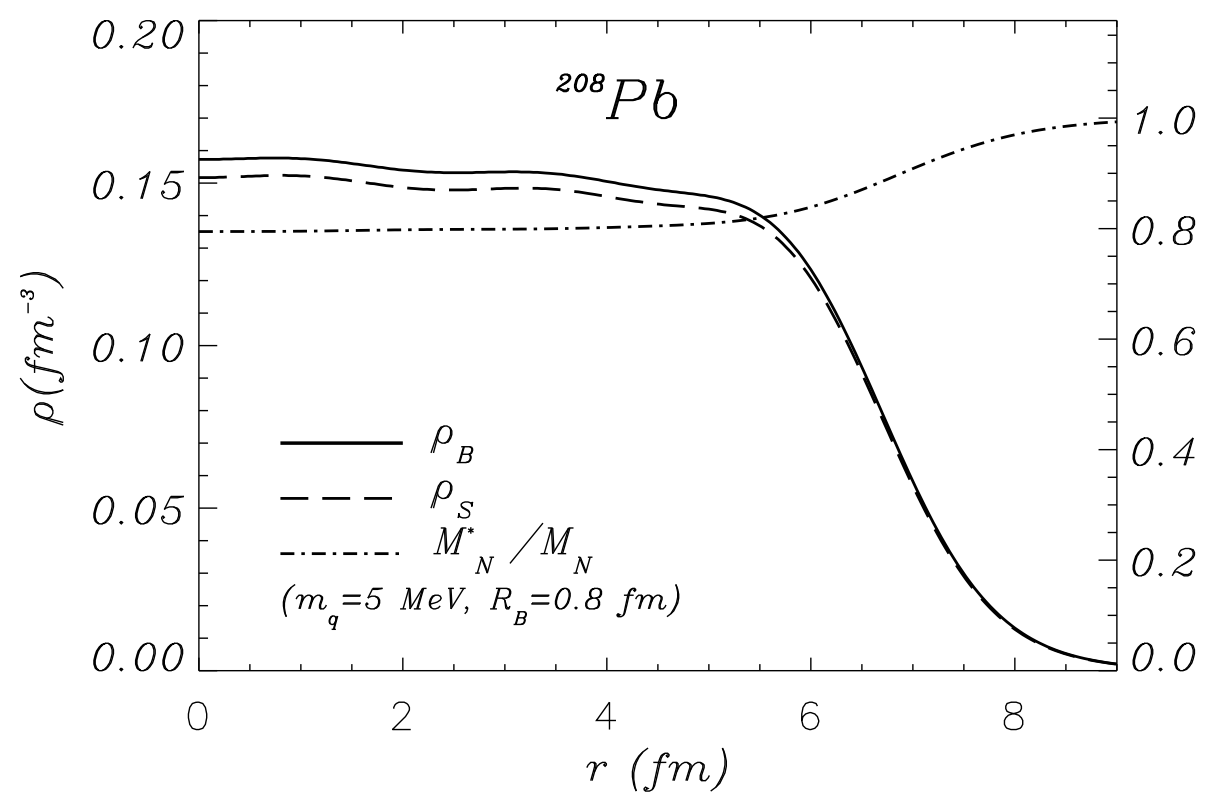

Figure 2: Model predictions for the effective nucleon mass, the baryon and scalar densities in ${ }^{208} \mathrm{~Pb}$ (for $m_{q}=5 \mathrm{MeV}$ and $R_{B}=0.8 \mathrm{fm}$ ). The scale on the right vertical axis is for $M_{N}^{\star} / M_{N}$.

In Fig.2, we show the baryon and scalar densities as well as the effective nucleon mass in lead. We expect that the baryon density in the interior of lead would be close to the saturation density of infinite nuclear matter. As seen in the figure, the calculated baryon density at the center is quite close to $0.15 \mathrm{fm}^{-1}$, which supports our choice of the parameters. 


\subsection{Comparison with experimental data}

First we show calculated charge density distributions, $\rho_{c h}$, in comparison with those of QHD [9, 25] and the experimental data in Figs.3-12. Having solved Eqs.(23) (29), we obtain the point-proton and neutron densities in a nucleus. In addition to those densities, we should include the effects of c.m. corrections, nucleon form factors, meson-exchange currents, etc. However, one knows that the dominant correction comes from the protonform factor [25]. We calculate the charge density as a convolution of the point-proton density, $\rho_{p}(\vec{r})$, with the proton charge distribution, $\rho_{c h}^{p}(\vec{r})$ :

$$
\rho_{c h}(\vec{r})=\int d \vec{r}^{\prime} \rho_{c h}^{p}\left(\vec{r}-\vec{r}^{\prime}\right) \rho_{p}\left(\vec{r}^{\prime}\right),
$$

where we have used a gaussian form for $\rho_{c h}^{p}$

$$
\rho_{c h}^{p}(\vec{r})=(\beta / \pi)^{3 / 2} \exp \left(-\beta r^{2}\right) .
$$

The parameter $\beta$, which determines the proton size, is chosen so as to reproduce the experimental rms charge radius of the proton, $0.82 \mathrm{fm}$ (i.e., $\beta=2.231 \mathrm{fm}^{-2}$ ). As we noted before, in the present model the rms radius of the nucleon in nuclear matter increases a little. However, this amount is quite small and it can be ignored in the numerical calculations of nuclear parameters.

The charge density distributions for ${ }^{40} \mathrm{Ca}$ (for $m_{q}=0,5,10 \mathrm{MeV}$ and $R_{B}=0.8 \mathrm{fm}$ ) are presented in Figs. 3-5. The experimental data is taken from Ref. [29]. Once the rms charge radius of ${ }^{40} \mathrm{Ca}$ is fitted by adjusting $m_{\sigma}$ the QMC model can reproduce $\rho_{c h}\left({ }^{40} \mathrm{Ca}\right)$ quite well. As seen in the figures, the calculated charge densities lie almost within the experimental (hatched) area. We note that the dependence of $\rho_{c h}\left({ }^{40} \mathrm{Ca}\right)$ on the bag radius is quite weak.

It is interesting to see the quantum oscillations of the interior density in lead. The dependence of $\rho_{c h}\left({ }^{208} \mathrm{~Pb}\right)$ on the quark mass is illustrated in Figs.6-8. We note that the dependence of $\rho_{c h}\left({ }^{208} \mathrm{~Pb}\right)$ on the free nucleon size is also quite weak. As seen in the figures, our model gives charge densities very close to those of QHD and still somewhat larger in the central region than those observed experimentally [30]. 


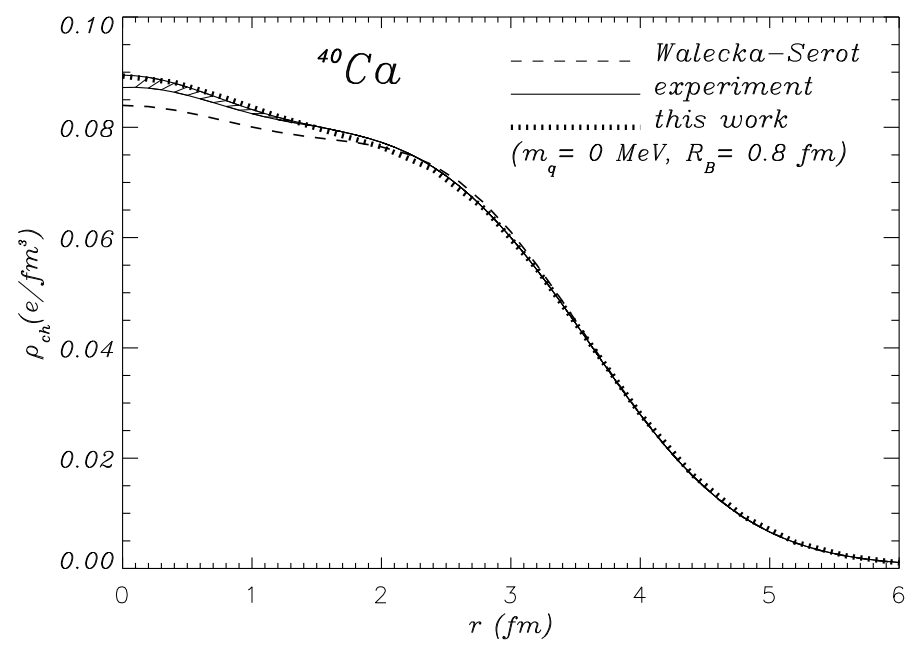

Figure 3: Charge density distribution for ${ }^{40} \mathrm{Ca}$ (for $m_{q}=0 \mathrm{MeV}$ and $R_{B}=0.8 \mathrm{fm}$ ) compared with the experimental data and that of QHD.

In Figs.9 and 10, we show respectively the charge density distributions for ${ }^{16} \mathrm{O}$ and ${ }^{90} \mathrm{Zr}$. For zirconium the calculated $\rho_{c h}$ lies in between that of the non-relativistic densitydependent Hartree-Fock calculations [3] and that of QHD. The experimental data for oxygen and zirconium are taken from Refs. [31] and [32]. (For both cases the dependence of $\rho_{c h}$ on $m_{q}$ and $R_{B}$ is weak.)

To see the isotope shift in charge density we have plotted $r^{2}$ times the difference between $\rho_{c h}\left({ }^{40} \mathrm{Ca}\right)$ and $\rho_{c h}\left({ }^{48} \mathrm{Ca}\right)$ in Figs.11 and 12. Its dependence on the bag radius is again weak for a small quark mass while it becomes a little stronger for $m_{q}=10 \mathrm{MeV}$, as shown in Fig.[12. The experimental data is taken from Ref.[33]. (Note that in this case we also checked that including the charge distribution of the neutron had a small effect.)

In Figs.13-15, we present the point-neutron density distributions, $\rho_{n}$, in calcium and lead. For ${ }^{40} \mathrm{Ca}$, since the dependence of $\rho_{n}$ on $m_{q}$ and $R_{B}$ is again fairly weak, only the result for $m_{q}=5 \mathrm{MeV}$ and $R_{B}=0.8 \mathrm{fm}$ is shown, together with the empirical fit [34] to proton scattering data. We again find reasonable agreement with the data. For the isotope shift of $\rho_{n}\left({ }^{48} \mathrm{Ca}\right)-\rho_{n}\left({ }^{40} \mathrm{Ca}\right)$, the calculated difference is closer to those of nonrelativistic results than to those of QHD. The neutron density distribution in lead is shown 


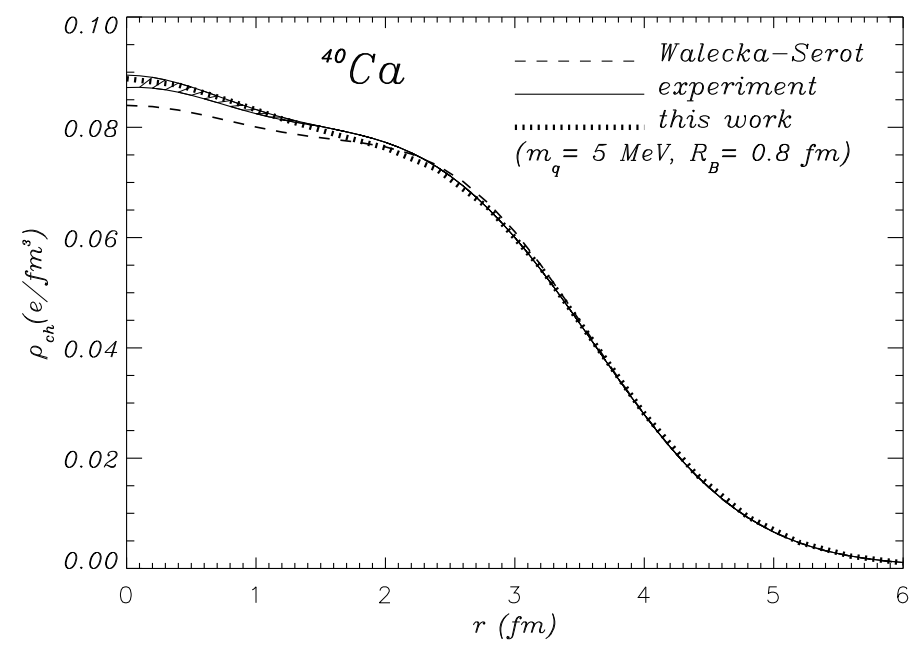

Figure 4: Same as Fig.5 (for $m_{q}=5 \mathrm{MeV}$ and $R_{B}=0.8 \mathrm{fm}$ ).

in Fig.15. Its behavior is again similar to that of QHD.

Table G gives a summary of the calculated binding energy per nucleon $(E / A)$, rms charge radii and the difference between nuclear rms radii for neutrons and protons $\left(r_{n}-r_{p}\right)$, for several closed-shell nuclei. Since the calculated properties do not depend strongly on $m_{q}$ and $R_{B}$, we only list the values for the QMC model with $m_{q}=5 \mathrm{MeV}$ and $R_{B}=$ $0.8 \mathrm{fm}$. References for the experimental values can be found in Ref. 255. While there are still some discrepancies between the results and data, the present model provides quite reasonable results. In particular, it gives much larger binding energies per nucleon than those of QHD while still reproducing the rms charge radii for medium and heavy nuclei quite well.

In Figs.16 and 17, the calculated spectra of ${ }^{40} \mathrm{Ca}$ and ${ }^{208} \mathrm{~Pb}$ are presented. Because of the relatively smaller scalar and vector fields in the present model than in QHD, the spin-orbit splittings are smaller. The improvement over QHD in the binding energy per nucleon comes at the expense of a reduction in the spin-orbit force. We should note that there is a strong correlation between the effective nucleon mass and the spin-orbit force. This problem is also discussed in Refs. [22, 23].

As a test of the sensitivity of the spin-orbit splitting to features of the model, we con- 




Figure 5: Same as Fig.5 (for $m_{q}=10 \mathrm{MeV}$ and $R_{B}=0.8 \mathrm{fm}$ ).

sider the case of a larger quark mass (noting that the Born-Oppenheimer approximation requires that the nucleon motion is relatively slow and the quarks are highly relativistic). For example, we have calculated the case $m_{q}=300 \mathrm{MeV}$ (and $R_{B}=0.8 \mathrm{fm}$ ) which is a typical constituent quark mass. The calculated spectra for $m_{q}=300 \mathrm{MeV}$ are also illustrated in Figs.16 and $17(\mathrm{QMCH})$. In this case, the various parameters were $g_{\sigma}^{2} / 4 \pi=$ $5.58, g_{\omega}^{2} / 4 \pi=8.51$ (to satisfy the saturation condition), $g_{\rho}^{2} / 4 \pi=6.45, m_{\sigma}=497 \mathrm{MeV}$ (to fit the rms charge radius of ${ }^{40} \mathrm{Ca}$ ), $K=334 \mathrm{MeV}$ and $M_{N}^{\star}=674 \mathrm{MeV}$ at saturation density. We should record that the bag radius in this case increases by $7 \%$ at saturation density (which is uncomfortably large). The slope parameter in Eq.(18) is $a=3.9 \times 10^{-4}$. One can expect that a heavy quark mass gives a spectrum closer to those of QHD[11, 21]. We can see from the figures that the calculated spectra are somewhat closer to the experimental data. We note that the charge density distributions for ${ }^{40} \mathrm{Ca}$ and ${ }^{208} \mathrm{~Pb}$ are also reproduced well in this case. 


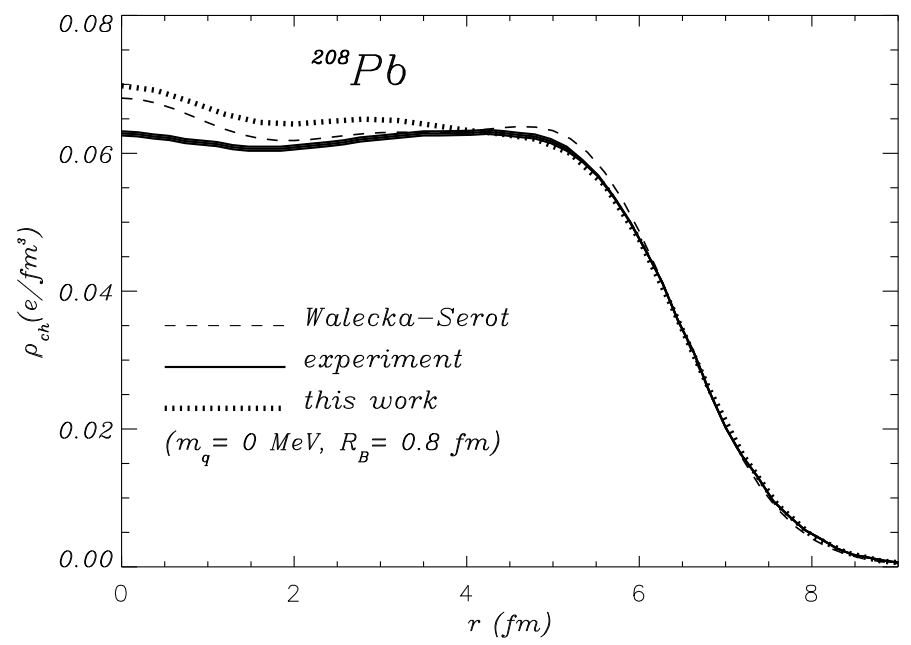

Figure 6: Charge density distribution for ${ }^{208} \mathrm{~Pb}$ (for $m_{q}=0 \mathrm{MeV}$ and $R_{B}=0.8 \mathrm{fm}$ ) compared with the experimental data and that of QHD.

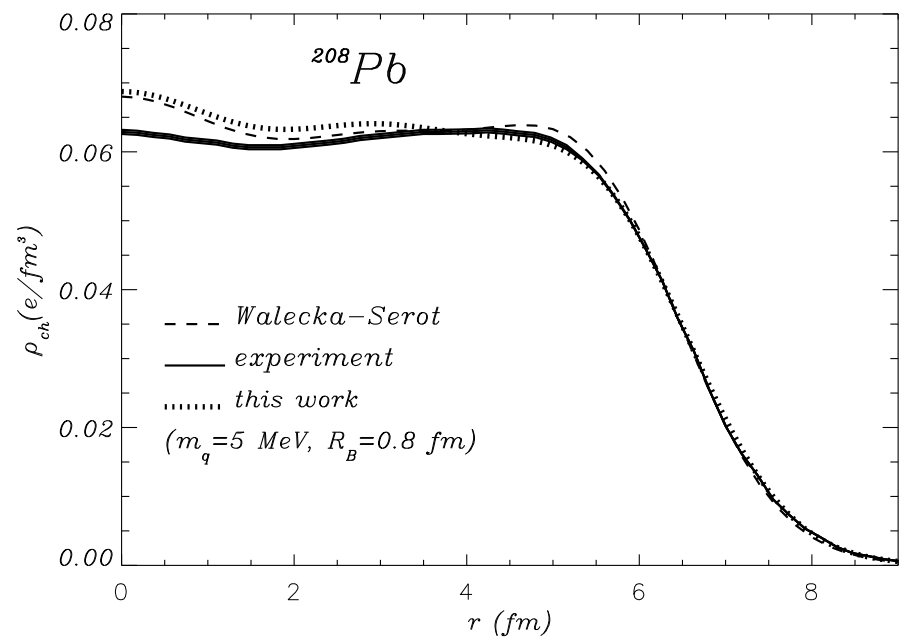

Figure 7: Same as Fig.8 (for $m_{q}=5 \mathrm{MeV}$ and $R_{B}=0.8 \mathrm{fm}$ ). 


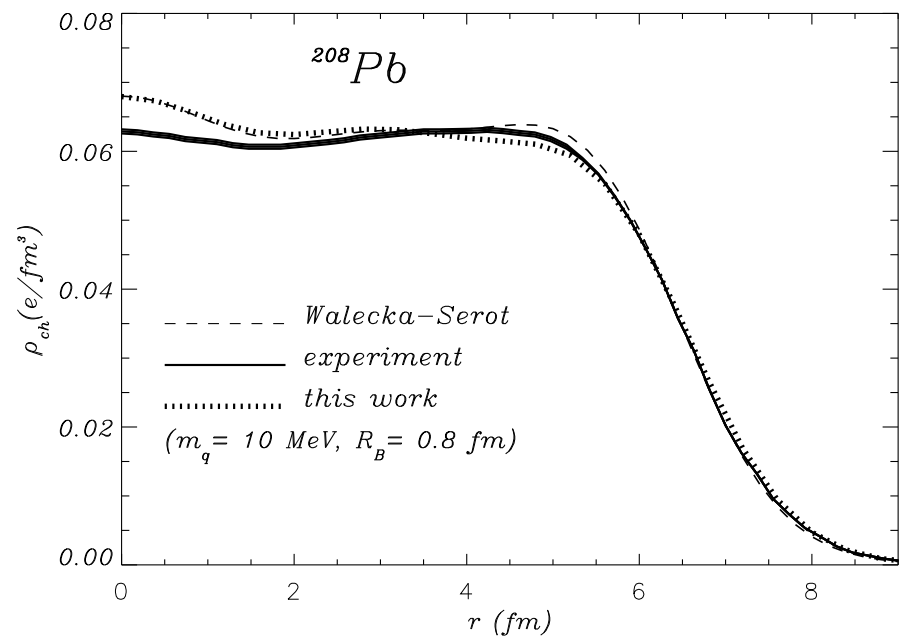

Figure 8: Same as Fig.8 (for $m_{q}=10 \mathrm{MeV}$ and $R_{B}=1.0 \mathrm{fm}$ ).

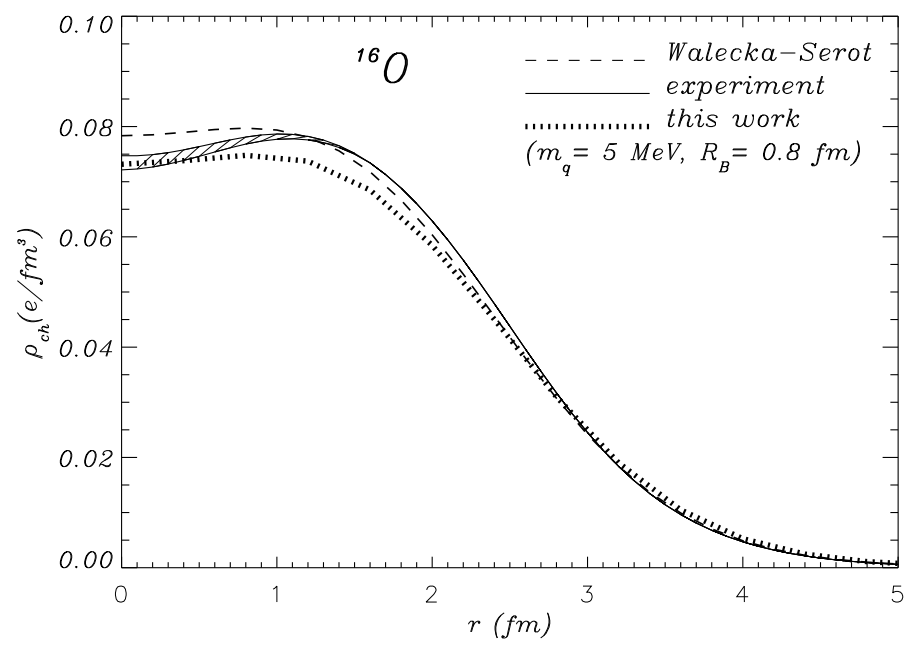

Figure 9: Charge density distribution for ${ }^{16} \mathrm{O}$ (for $m_{q}=5 \mathrm{MeV}$ and $R_{B}=0.8 \mathrm{fm}$ ) compared with the experimental data and that of QHD. 


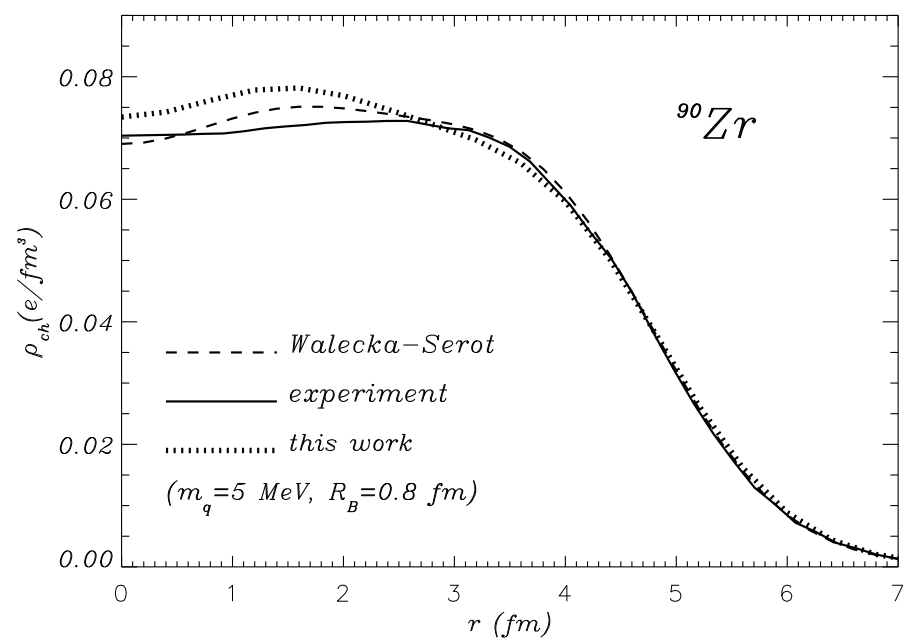

Figure 10: Charge density distribution for ${ }^{90} \mathrm{Zr}$ (for $m_{q}=5 \mathrm{MeV}$ and $R_{B}=0.8 \mathrm{fm}$ ) compared with the experimental data and that of QHD.

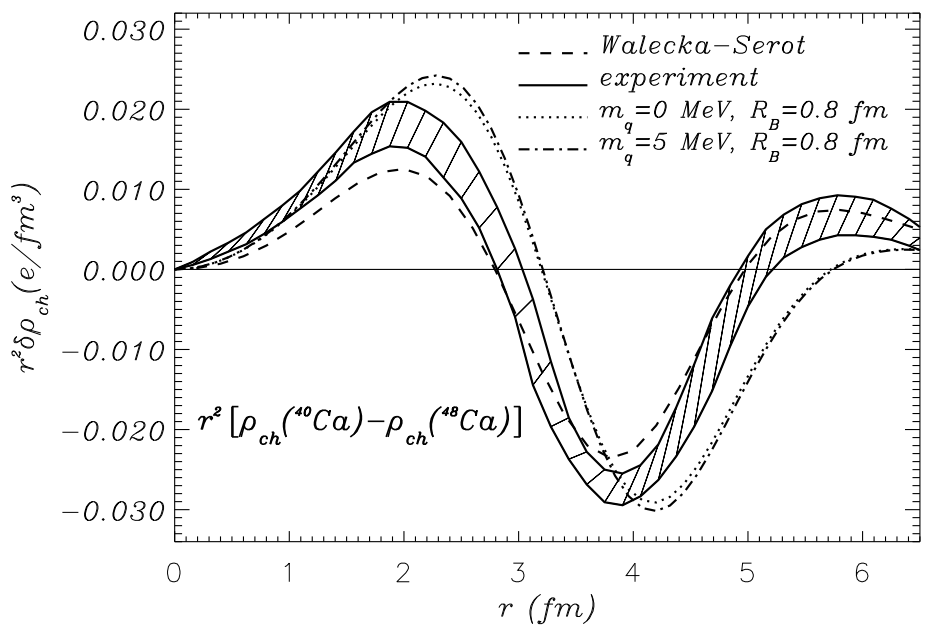

Figure 11: Isotope shift between $\rho_{c h}\left({ }^{40} \mathrm{Ca}\right)$ and $\rho_{c h}\left({ }^{48} \mathrm{Ca}\right)$ compared with the experimental data and that of QHD (for $m_{q}=0$ and $5 \mathrm{MeV}$ with $R_{B}=0.8 \mathrm{fm}$ ). 


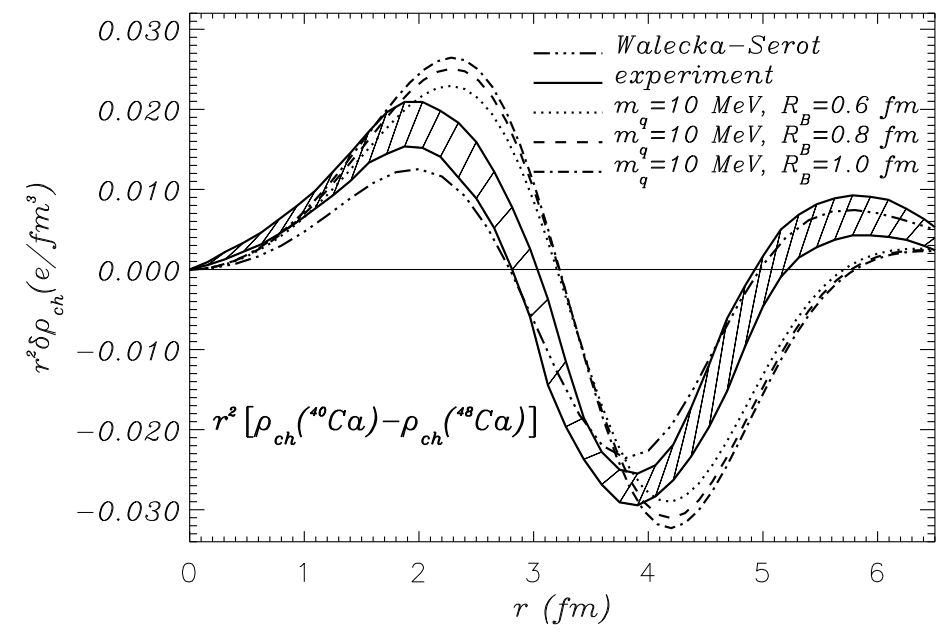

Figure 12: Same as Fig.13 (for $m_{q}=10$ with $R_{B}=0.6,0.8$ and $1.0 \mathrm{fm}$ ).

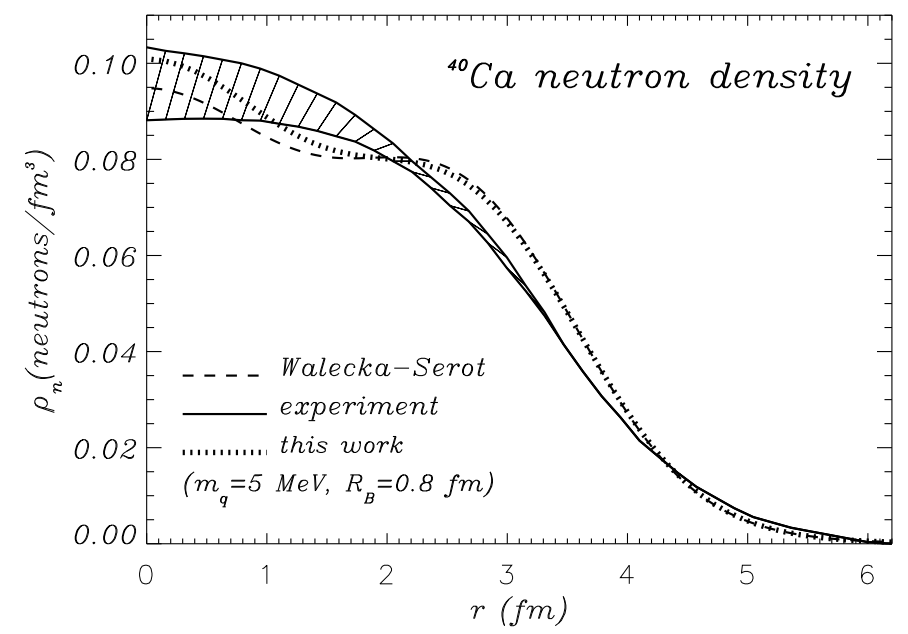

Figure 13: Point-neutron density distribution in ${ }^{40} \mathrm{Ca}$ (for $m_{q}=5 \mathrm{MeV}$ and $R_{B}=0.8$ fm) compared with that of QHD and the empirical fit. 


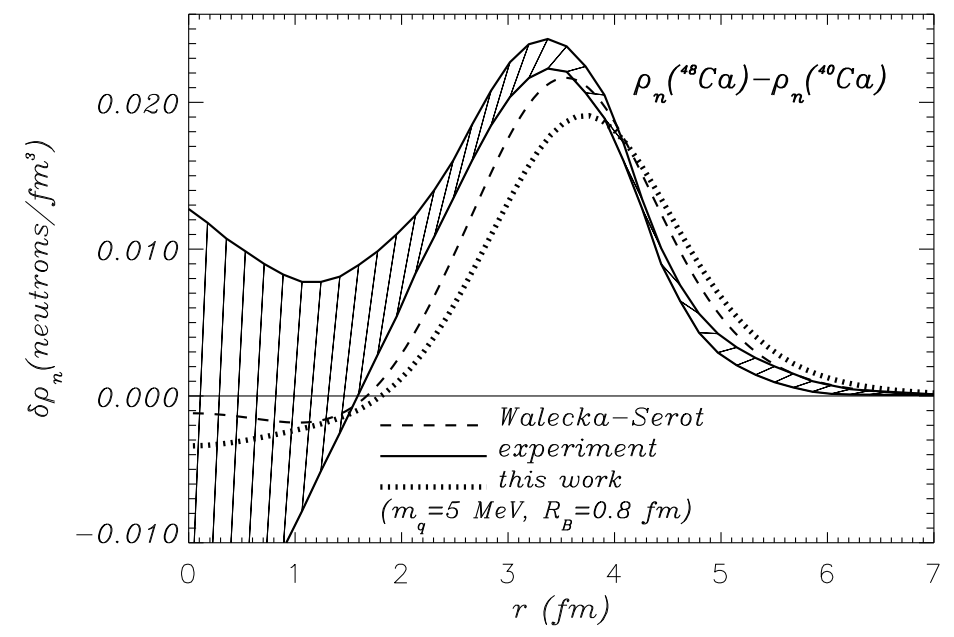

Figure 14: Difference between $\rho_{n}\left({ }^{48} \mathrm{Ca}\right)$ and $\rho_{n}\left({ }^{40} \mathrm{Ca}\right)$ compared with that of QHD and the empirical fit (for $m_{q}=5$ and $R_{B}=0.8 \mathrm{fm}$ ).



Figure 15: Point-neutron density distribution in ${ }^{208} \mathrm{~Pb}$ (for $m_{q}=5 \mathrm{MeV}$ and $R_{B}=0.8$ fm) compared with that of QHD and the empirical fit. 
Table 4: Binding energy per nucleon $E / A$ (in $\mathrm{MeV}$ ), rms charge radius $r_{c h}$ (in fm) and difference between $r_{n}$ and $r_{p}$ (in fm). $m_{q}=5 \mathrm{MeV}$ and $R_{B}=0.8 \mathrm{fm}$. ( ${ }^{*}$ fit)

\begin{tabular}{c|ccc|ccc|ccc}
\hline & \multicolumn{3}{|c|}{$-E / A$} & \multicolumn{3}{c|}{$r_{c h}$} & \multicolumn{3}{c}{$r_{n}-r_{p}$} \\
\hline Model & QMC & QHD & Exp. & QMC & QHD & Exp. & QMC & QHD & Exp. \\
\hline${ }^{16} \mathrm{O}$ & 5.84 & 4.89 & 7.98 & 2.79 & 2.75 & 2.73 & -0.03 & -0.03 & 0.0 \\
${ }^{40} \mathrm{Ca}$ & 7.36 & 6.31 & 8.45 & $3.48^{*}$ & $3.48^{*}$ & 3.48 & -0.05 & -0.06 & $0.05 \pm 0.05$ \\
${ }^{48} \mathrm{Ca}$ & 7.26 & 6.72 & 8.57 & 3.52 & 3.47 & 3.47 & 0.23 & 0.21 & $0.2 \pm 0.05$ \\
${ }^{90} \mathrm{Zr}$ & 7.79 & 7.02 & 8.66 & 4.27 & 4.26 & 4.27 & 0.11 & 0.10 & $0.05 \pm 0.1$ \\
${ }^{208} \mathrm{~Pb}$ & 7.25 & 6.57 & 7.86 & 5.49 & 5.46 & 5.50 & 0.26 & 0.27 & $0.16 \pm 0.05$ \\
\hline
\end{tabular}






Figure 16: Model predictions for the energy spectrum for ${ }^{40} \mathrm{Ca}$. QMC(H) denotes the case for $m_{q}=5(300) \mathrm{MeV}$ and $R_{B}=0.8 \mathrm{fm}$. 


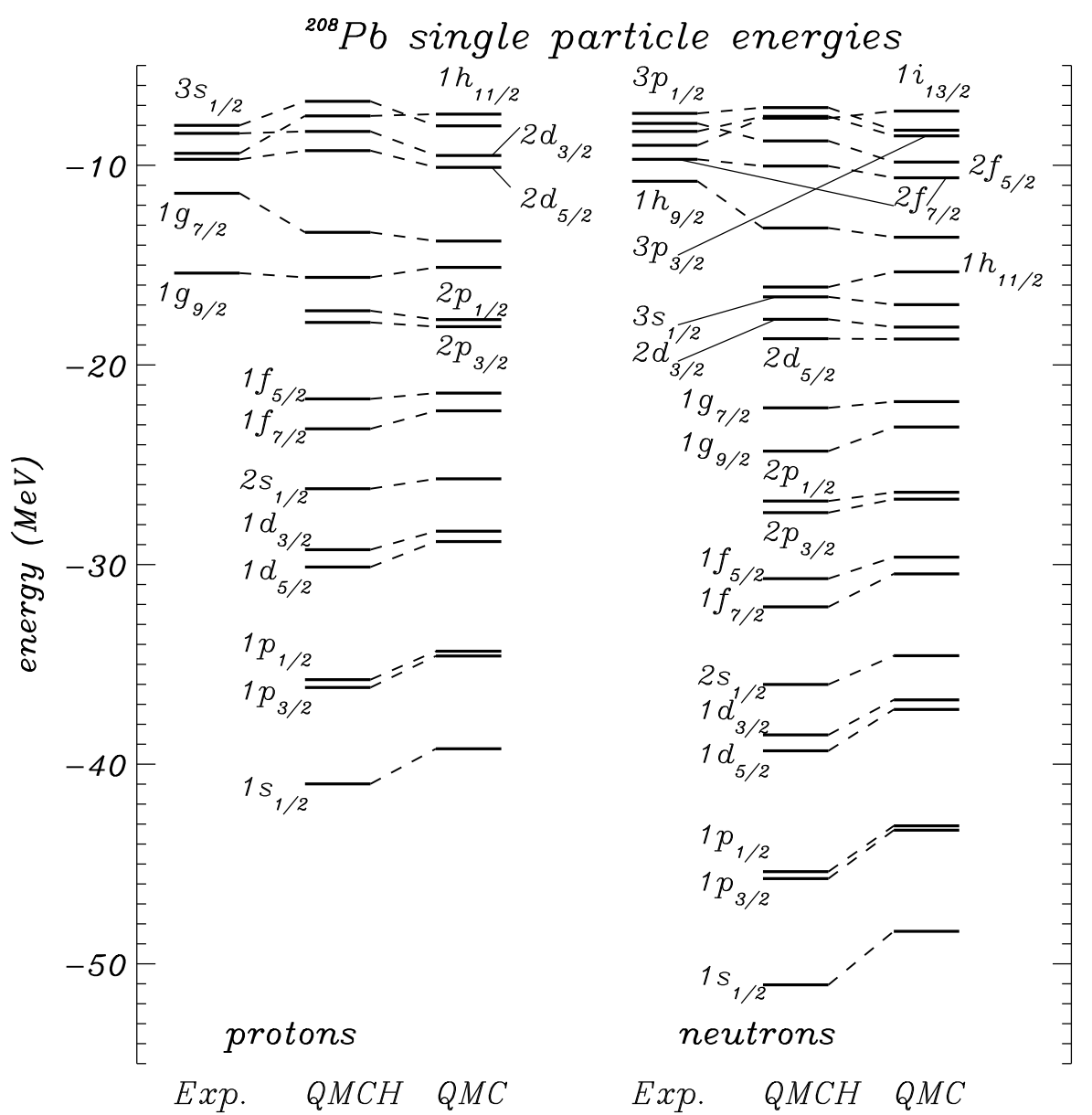

Figure 17: Same as Fig.18 (for ${ }^{208} \mathrm{~Pb}$ ). 


\section{Summary, discussion and further applications}

Starting with the quark-meson coupling model, in which quarks confined in nucleon bags interact through the exchange of scalar and vector mesons, we have presented a generalisation of QHD with a $\sigma$ field-dependent scalar coupling to describe finite nuclei quantitatively. The physical origin of this field-dependence, which provides a new saturation mechanism for nuclear matter, is the relatively rapid increase of the lower Dirac component of the wavefunction of the confined, light quark. We have then derived a set of coupled non-linear differential equations which must be solved self-consistently but which are not much more difficult to solve than the relativistic Hartree equations of QHD. Our calculations for static, closed-shell nuclei from ${ }^{16} \mathrm{O}$ to ${ }^{208} \mathrm{~Pb}$ reproduce fairly well the observed charge density distributions, neutron density distributions etc.

It will be very interesting to explore the connection between the $\sigma$ field-dependence of the variation of the effective $\sigma-\mathrm{N}$ coupling constant, which arises so naturally here, and the variation found empirically in earlier work. We note, in particular, that while our numerical results depend on the particular model chosen here (namely, the MIT bag model), the qualitative features which we find (such as the $\sigma$ field-dependent decrease of the scalar coupling etc.) will apply in any model in which the nucleon contains light quarks and the attractive $\mathrm{N}-\mathrm{N}$ force is a Lorentz scalar. Of course, it will be important to investigate the degree of variation in the numerical results for other models of nucleon structure 23.

In the present model there are, however, still some discrepancies in energy spectra of nuclei, in particular, the spin-orbit splittings. In the previous section we have briefly discussed one possibility to partly overcome this defect in the present model. As an alternative approach, Jin and Jennings 22] and Blunden and Miller[23] have considered variations of the bag constant $B$ and $z$ parameter in nuclear matter, which have been suggested by the fact that quarks are presumably deconfined at high enough densities. This was taken to suggest that $B$ might decrease with increasing density. Adopting this idea, Blunden and Miller 23 have studied properties of both nuclear matter and finite 
nuclei. In their approach, $B$ in matter, $B^{\star}$, is given by

$$
B^{\star}=B\left[1-\alpha_{B} \frac{U_{s}(\vec{r})}{M_{N}}\right],
$$

where $U_{s}$ is an average scalar potential and $\alpha_{B}$ is an arbitrary parameter. For finite nuclei the results move towards those of $\mathrm{QHD}$, and there is an improvement in the spin-orbit splittings. However, the nuclear incompressibility, $K$, is again larger than the experimental data, and the bag radius at saturation density increases by $5 \% \sim 13 \%$ [23] or more 22], which seems uncomfortably large [1]. We probably ought to construct a formalism beyond the Hartree approximation for this class of models in the future.

In this work we have not considered any effect of a decrease of the meson masses in nuclear matter. (In Ref.[19] these effects were investigated in infinite nuclear matter, using an earlier version of the model.) The $\sigma$-meson mass which we found it necessary to use in this paper ranges from $400 \sim 450 \mathrm{MeV}$. It seems rather small compared with the empirical $\sigma$-mass in the OBEP analyses of the free NN force [2, 28, 35]. On the other hand, noting its origin in two-pion-exchange with $\mathrm{N}-\Delta$ intermediate states, we observe that the N- $\Delta$ mass difference would decrease in medium by a similar percentage. It will be interesting, in future work, to incorporate a self-consistent reduction of the vector-meson mass [19] (as well as the scalar-meson mass 36]) into the calculation of the properties of finite nuclei.

The successful generalisation of the QMC model to finite nuclei opens a tremendous number of opportunities for further work. For example, earlier results for the OkamotoNolen-Schiffer anomaly[13], the nuclear EMC effect[20], the charge-symmetry violating correction to super-allowed Fermi beta-decay 15 and so on, can now be treated in a truly quantitative way. Since the present model can provide self-consistent quark wave functions in a deeply bound nucleon, e.g., $1 s_{1 / 2}$ in ${ }^{208} \mathrm{~Pb}$, it would also be very interesting to investigate variations in magnetic moments, $g_{A}$ and so on, for such nucleons [37].

For hypernuclei the natural extension (see Ref.[19) is to assume that the $\sigma$ and $\omega$ mesons couple only to the non-strange constituents. From our discussion of the spin-orbit force in Ref. 21] and the fact that the spin of the $\Lambda$ is carried entirely by the strange 
quark, one can easily see that the $\Lambda$ spin-orbit force will arise entirely from the Thomas precession term. This means that the $\Lambda$ spin-orbit force is very naturally suppressed in this model - as observed experimentally. It will be important to follow this observation with quantitative results.

Finally, we list a number of important ways in which this model could be extended: replacing the MIT bag by a model respecting PCAC (e.g., the cloudy bag model[38]); replacing $\sigma$-exchange by two-pion exchange; replacing $\omega$ exchange by nucleon overlap at short distance, and so on. In terms of further theoretical development it will also be interesting to compare the present model with more phenomenological, non-linear extensions of QHD - as reviewed recently in Ref.[39]. On the practical side, we stress that the present model can be applied to all the problems for which QHD has proven so attractive, with very little extra effort.

\section{Acknowledgements}

The authors are pleased to thank Pierre A.M. Guichon for valuable discussions and comments. This work was supported by the Australian Research Council. 


\section{References}

[1] D.F. Geesaman, K. Saito and A.W. Thomas, Annu. Rev. Nucl. Part. Sci. 45 (1995) 337 ;

M. Arneodo, Phys. Rep. 240 (1994) 301.

[2] K. Holinde and A.W. Thomas, Phys. Rev. C42 (1990) R1195;

S. Deister et al., Few Body Systems 10 (1991) 1;

J. Haidenbauer, K. Holinde and A.W. Thomas, Phys. Rev. C49 (1994) 2331.

[3] J.W. Negele, Phys. Rev. C1 (1970) 1260;

X. Campi and D.W.L. Sprung, Nucl. Phys. A194 (1972) 401;

J.L. Friar and J.W. Negele, Advan. Nucl. Phys. 8 (1975) 219.

[4] K. Okamoto, Phys. Lett. 11 (1964) 150;

J.A. Nolen, Jr. and J.P. Schiffer, Annu. Rev. Nucl. Sci. 19 (1969) 471.

[5] S. Shlomo, Rep. Prog. Phys. 41 (1978) 957;

G.A. Miller, B.M.K. Nefkens and I. Šlaus, Phys. Rep. 194 (1990) 1.

[6] H. Sato, Nucl. Phys. A296 (1976) 378.

[7] T. Goldman, J.A. Henderson and A.W. Thomas, Few Body Systems 12 (1992) 123;

G. Krein, A.W. Thomas and A.W. Williams, Phys. Lett. B317 (1993) 293;

J. Piekarewicz and A.W. Williams, Phys. Rev. C47 (1993) R2461;

T. Hatsuda, E.M. Henley, Th. Meissner and G. Krein, Phys. Rev. C49 (1994) 452.

[8] D.H. Wilkinson, Nucl. Phys. A571 (1994) 87; Z. Phys. A348 (1994) 129;

I.S. Towner and J.C. Hardy, in The Nucleus as a Laboratory for Studying Symmetries and Fundamental Interaction, ed. by E.M. Henley and W.C. Haxton, (nucl-th/9504015) (World Scientific, 1996);

G. Savard et al., Phys. Rev. Lett. 74 (1995) 1521. 
[9] J.D. Walecka, Ann. Phys. (N.Y.) 83 (1974) 491;

B.D. Serot and J.D. Walecka, Advan. Nucl. Phys. 16 (1986) 1.

[10] P.A.M. Guichon, Phys. Lett. B200 (1988) 235.

[11] K. Saito and A.W. Thomas, Phys. Lett. B327 (1994) 9;

Phys. Rev. C52 (1995) 2789.

[12] S. Fleck et al., Nucl. Phys. A510 (1990) 731;

V.K. Mishra et al., Phys. Rev. C46 (1992) 1143;

M.K. Banerjee, Phys. Rev. C45 (1992) 1359;

E. Naar and M.C. Birse, J. Phys. G19 (1993) 555.

[13] K. Saito and A.W. Thomas, Phys. Lett. B335 (1994) 17.

[14] A.W. Thomas and K. Saito, "The Role of Nucleon Structure in Nuclear Physics", in Proc. Int. Conf. on Physics with GeV Beams, eds. H. Machner and K. Sistemich, World Scientific, Singapore (1995) p.175.

[15] K. Saito and A.W. Thomas, Phys. Lett. B363 (1995) 157.

[16] A.W. Thomas and K. Saito, "Charge Symmetry Violation in Nuclear Physics", in Proc. of the Int. Conf. on Weak and Electromagnetic Interactions in Nuclei (WEIN '95), eds. H. Ejiri, T. Kishimoto and T. Sato, World Scientific, Singapore (1995) p.495.

[17] G.Q. Li, C.M. Ko and G.E. Brown, Phys. Rev. Lett. 75 (1995) 4007.

[18] G. Agakichiev et al. (CERES Collaboration), Phys. Rev. Lett. 75 (1995) 1272; M. Masera et al. (HELIOS-3 Collaboration), Nucl. Phys. A590 (1995) 93c.

[19] K. Saito and A.W. Thomas, Phys. Rev. C51 (1995) 2757.

[20] K. Saito, A. Michels and A.W. Thomas, Phys. Rev. C46 (1992) R2149; A.W. Thomas, K. Saito and A. Michels, Aust. J. Phys. 46 (1993) 3; 
K. Saito and A.W. Thomas, Nucl. Phys. A574 (1994) 659.

[21] P.A.M. Guichon, K. Saito, E. Rodionov and A.W. Thomas, nucl-th/9509034, to appear in Nucl. Phys. A (1996);

P.A.M. Guichon, K. Saito and A.W. Thomas, nucl-th/9602022, to appear in Australian Journal of Physics (1996).

[22] Xuemin Jin and B.K. Jennings, Phys. Lett. B374 (1996) 13;

TRIUMF preprint, TRI-PP-96-8 (nucl-th/9604018).

[23] P.G. Blunden and G.A. Miller, nucl-th/9602031 (1996), to appear in Phys. Rev. C (1996).

[24] C. Fuchs, H. Lenske and H.H. Wolter, Phys. Rev. C52 (1995) 3043;

H. Lenske and C. Fuchs, Phys. Lett. B345 (1995) 355.

[25] C.J. Horowitz and B.D. Serot, Nucl. Phys. A368 (1981) 503.

[26] C.J. Horowitz, D.P. Murdoch and B.D. Serot, Computational Nuclear Physics 1, eds. K. Langanke, J.A. Maruhn and S.E. Koonin (Springer-Verlag, Berlin, 1991), p.129.

[27] B.B.P. Sinha et al., Phys. Lett. B53 (1971) 217.

[28] R. Machleidt, Advan. in Nucl. Phys. 19 (1989) 189.

[29] I. Sick et al., Phys. Lett. B88 (1979) 245.

[30] B. Frois et al., Phys. Rev. Lett. 38 (1977) 152.

[31] I. Sick and J.S. McCarthy, Nucl. Phys. A150 (1970) 631.

[32] L.A. Fajardo et al., Phys. Lett. B37 (1971) 363.

[33] B. Frois, Lecture notes in Physics 108 (1979) p.52. 
[34] L. Ray, Phys. Rev. C19 (1979) 1855;

L. Ray and P.E. Hodgson, Phys. Rev. C20 (1979) 2403.

[35] K. Erkelenz, Phys. Rep. C13 (1974) 194.

[36] K. Saito, T. Maruyama and K. Soutome, Phys. Rev. C40 (1989) 407;

T. Hatsuda and T. Kunihiro, Phys. Rep. 247 (1994) 221.

[37] T. Yamazaki, Mesons in Nuclei, vol.II, eds. M. Rho and D.H. Wilkinson (North Holland, Amsterdam, 1979), p.651.

[38] S. Théberge et al., Phys. Rev. D22 (1980) 2838; D23 (1981) 2106(E);

A. W. Thomas, Adv. Nucl. Phys. 13 (1984) 1;

G. A. Miller, in Int. Rev. Nucl. Phys. 2 (1984).

[39] A. Delfino et al., nucl-th/9602004 (1996). 